\title{
The strength of coughing may forecast the likelihood of spread of multi-drug resistant microorganisms from the respiratory tract of colonized patients
}

\author{
Magda Diab-Elschahawi ${ }^{1}$, Luigi Segagni Lusignani ${ }^{1}$, Peter Starzengruber ${ }^{1}$, Dieter Mitteregger ${ }^{2}$, Andrea Wagner $^{1}$, \\ Ojan Assadian ${ }^{1^{*}}$ and Elisabeth Presterl ${ }^{1}$
}

\begin{abstract}
Background: Current recommendations indicate that patients who are coughing and have multidrug resistant microorganisms (MDROs) in their sputum are considered to be shedders and should be cared for in single room isolation at least until symptoms resolve. Airborne spread and subsequent contamination of surfaces adjacent to patients may contribute to transmission. Hence, isolation measures for patients colonized or infected with MDRO at their respiratory tract are intended to interrupt such transmission. However, the potential for microbial shedding in patients with MDRO-positive microbiological reports from their respiratory tract and factors justifying the need for single room isolation are viewed controversially.
\end{abstract}

Methods: Cough aerosol produced by patients colonized with MDROs was measured for viable counts. Descriptive analysis together with logistic regression analysis was performed to assess the impact of strength of cough on growth of MDRO on culture plates.

Results: In 18\% (23/128) MDRO were transmitted. Multivariate analysis revealed that strength of cough significantly predicts the yield of MDRO on culture plates $(P=0.012)$.

Conclusion: Based on these results it can be concluded that risk stratification for decision of single room isolation of patients colonized or infected with MDROs at their respiratory tract may also take the severity of cough into consideration. However, more work is required in order to assess the severity of cough objectively.

Keywords: Multidrug resistant microorganism, Cough, Aerogene spread, Risk factors, Single room isolation, Infection Control

\section{Background}

Multidrug resistant microorganisms (MDROs) may be transmitted by different routes, including blood borne, droplet, airborne and contact transmission. In general, and for all health care settings, adherence to hand hygiene practices may have the highest impact on prevention of direct and indirect contact transmission [1]. However, airborne spread and subsequent contamination

\footnotetext{
* Correspondence: ojan.assadian@meduniwien.ac.at

'Department of Hospital Hygiene and Infection Control, Vienna General Hospital, Medical University Vienna, Waehringer Guertel 18-20, 1090 Vienna, Austria
}

Full list of author information is available at the end of the article of surfaces adjacent to patients may also contribute to transmission [2-5]. Isolation measures for patients colonized or infected with MDRO at their respiratory tract are intended to interrupt such transmission. Current recommendations $[6,7]$ indicate that patients who are coughing and have MDRO in their sputum are considered to be shedders and should be cared for in single room isolation at least until symptoms resolved. However, in many healthcare facilities the availability of single room isolation is limited, and the automatic and prolonged use has been questioned [8]. Information on the ability of spreading MDRO through coughing is scant and risk factors to identify those patients who shall 
be considered as relevant aerogene shedders of MDRO are unknown.

The aim of this study was to determine the potential for microbial shedding in patients with an MDROpositive microbiological report from their respiratory tract and to identify factors justifying the need for single room isolation.

\section{Methods}

\section{Inclusion criteria and specimen collection}

During a 1- year period all patients older than 18 years admitted to the University hospital of Vienna (VUH) whose respiratory tract was found to be colonized or infected with methicillin-resistant Staphylococcus aureus (MRSA), extended-spectrum beta-lactamase producing gram-negative bacteria (ESBL) including Escherichia coli or Klebsiella pneumoniae, or vancomycin resistant enterococci (VRE) were included. Subjects were asked to cough onto two culture plates after taking a maximal inspiration, i.e. Columbia blood agar with 5\% sheep blood (Becton Dickinson GmbH, Heidelberg, Germany) and depending on the nature of the colonizing organism a selective agar plate: chromID ${ }^{\circ}$ S. aureus, chromID ${ }^{\circ}$ ESBL, chromID ${ }^{\circ}$ VRE, bioMérieux, Marcy l'Etoile, France). For all samples, the same infection control practitioner positioned culture plates $5 \mathrm{~cm}$ in front of the patient's mouth. The strength of cough was denoted as "strong $(++)$ " or "weak $(+)$. Participants gave written informed consent. The Ethics Review Committee of The Medical University of Vienna approved the study (EC No. 1140/2012).

Specimens were labelled and immediately transferred to the microbiology laboratory for further analysis. Culture plates from all specimens were incubated at $37^{\circ} \mathrm{C}$ and examined after 24 and $48 \mathrm{~h}$. All possibly significant isolates were identified to species level using the specific colony coloration of the chromogenic medium while in case of growth limited to the blood agar plate or in case of ambiguous green-coloured colonies on the chromID ${ }^{\circ}$ ESBL by MALDI-TOF mass spectrometry (Bruker Daltonik GmbH, Bremen, Germany). Appropriate resistance testing according to current EUCAST recommendations (www.eucast.org) was performed in order to confirm the drug resistance status.

\section{Statistical analysis}

Statistical analysis was performed using SPSS software, version 20.0. Beyond descriptive analysis, a logistic regression analysis was performed to assess the impact of the independent variables (age, gender, species of $\mathrm{MDRO}$, strength of cough) on the dependent variable (growth of MDRO on culture plate) using 95\% CI and adjusted odds ratio (AOR). A P-value of less than 0.05 was considered to be statistically significant.

\section{Results and discussion}

A total of 128 cough episodes were analysed. Demographic data and clinical information are summarized in Table 1. In total, $18 \%(23 / 128)$ of patients colonized or infected with MDRO transmitted the organisms from their respiratory tract onto the culture plates by coughing. Multivariate logistic regression analysis (Table 2) revealed that presence or absence of MDRO on culture plates was significantly associated with strength of cough $(P=0.012)$, but not with patients' age $(P=0.593)$ or gender $(P=0.148)$, or the microbial species of the investigated MDRO ( $\mathrm{P}=0.523)$.

Retrieval or absence of MRSA on culture plates as compared to other MDROs was not statistically significant $(P=0.955)$, however, the strength of coughing correlated significantly with MRSA retrieval $(\mathrm{P}=0.014)$. The same observation was found for $\operatorname{VRE}(\mathrm{P}=0.275$ and $\mathrm{P}=0.015$, respectively), and ESBL $(\mathrm{P}=0.266$ and $\mathrm{P}=0.010$, respectively). The stratified subset analyses for the investigated MDROs confirmed the observation made for MDROs in general, supporting the result that strength of coughing, but not the respective species, is associated with shedding or non-shedding.

In our institution, the current practice for single room isolation of patients colonized or infected with MDRO is based on a structured risk assessment strategy. Depending on an individual assessment which considers the possibility for transmitting microorganisms on basis of their anatomic location, patients may fall into one of three categories: (a) no isolation, (b) contact isolation or

Table 1 Demographic data and clinical characteristics of the study population

\begin{tabular}{|c|c|c|c|}
\hline \multirow[b]{2}{*}{ Variables } & \multicolumn{2}{|c|}{$\begin{array}{l}\text { Cough plates- outcome } \\
\text { (growth/no growth) }\end{array}$} & \multirow{2}{*}{$\begin{array}{l}\text { Total } n=128 \\
n(\%)\end{array}$} \\
\hline & $\begin{array}{l}\text { growth } \\
(n=23)\end{array}$ & $\begin{array}{l}\text { no growth } \\
(n=105)\end{array}$ & \\
\hline \multicolumn{4}{|l|}{ Gender } \\
\hline Male & 14 & 52 & 66 (51.6) \\
\hline Female & 9 & 53 & $62(48.4)$ \\
\hline \multicolumn{4}{|l|}{ Age } \\
\hline Median (IQR) & \multicolumn{2}{|c|}{66 (53-72) years } & \\
\hline$\leq 65$ years & 13 & 50 & $63(49.2)$ \\
\hline$>65$ years & 10 & 55 & $65(50.8)$ \\
\hline \multicolumn{4}{|c|}{ Microorganism } \\
\hline MRSA & 10 & 41 & $51(39.8)$ \\
\hline VRE & 4 & 31 & $35(27.3)$ \\
\hline ESBL & 9 & 33 & $42(32.8)$ \\
\hline \multicolumn{4}{|c|}{ Strength of cough } \\
\hline Strong & 21 & 67 & $88(68.8)$ \\
\hline Weak & 2 & 38 & $40(31.3)$ \\
\hline
\end{tabular}




\begin{tabular}{|c|c|c|c|}
\hline \multirow[b]{2}{*}{ Independent variables } & \multicolumn{3}{|c|}{ MDRO growth on agar-plate (yes/no) } \\
\hline & $\begin{array}{l}\text { Adjusted odds } \\
\text { ratio (AOR) }\end{array}$ & $95 \% \mathrm{Cl}$ & P-value \\
\hline Age & 1.295 & $0.501-3.346$ & 0.593 \\
\hline Gender & 2.038 & $0.776-5.353$ & 0.148 \\
\hline Any Species of MDRO & 0.834 & $0.477-1.457$ & 0.523 \\
\hline Strength of cough & 7.336 & $1.558-34.537$ & 0.012 \\
\hline
\end{tabular}

$P$ - value calculated by multiple logistic regression analysis.

(c) strict isolation in a single room. For patients colonized with MDRO at their respiratory tract, as indicated by positive microbiological yield from sputum, tracheal secret, pharynx or anterior nares, single room isolation will be required, provided the patient is not ventilated with a closed breathing circuit. So far, there is little data available to decide if such patients may be epidemiologically relevant aerogene shedders of MDROs, since the role of droplet transmission in MDRO spread is not sufficiently investigated [5,7,9]. However, although this approached may be proactive and provides a safe environment of care for other patients, the benefits of single room isolation of patients colonized in their respiratory tract or their cohorting for reducing the spread of MDRO are discussed controversially [8]. Wigglesworth et al. [10] concluded that either isolation capacity needs to be increased or evidence-based risk assessment shall be applied in situations where isolation demands exceed availability.

Our study demonstrates that about every fifth patient colonized in the respiratory tract will transfer viable MDROs to close surfaces during coughing. However, a multivariate analysis reviles that transmission is seven times (AOR: 7.33) more likely if the patients coughs strongly. Based on these results it could be suggested that strict single room isolation may not be necessary in all patients colonized or infected with MRDOs at their respiratory tract. Aside of the anatomic location of microorganisms, the patient's compliance to follow standard infection control measures, and his mobility, the severity of cough may potentially be a further aspect which could be used for an individual risk-assessment in order to decide for a single room isolation.

Our study has a number of limitations. The assessment of cough intensity was based on subjective judgement and differentiated only "strong" and "weak". Although the same infection control practitioner assessed all cough intensities, and misclassification of cough intensities due to different observers was reduced to a minimum, a standardized method of measurement allowing accurate interpretation would be an advantage for future studies. A second limitation is that environmental surfaces $>5 \mathrm{~cm}$ from the patient were not sampled in parallel for presence or absence of MDRO. Therefore, the presented results do not allow statements on how far MDROs can be spread when a patient is coughing.

\section{Conclusion}

Based on the present results it can be concluded that risk stratification for decision of single room isolation of patients colonized or infected with MDROs at their respiratory tract may also take the severity of cough into consideration. However, more work is required in order to assess the severity of cough objectively.

\section{Abbreviations}

AOR: Adjusted odds ration; Cl: Confidence Interval; ESBL: Extended-spectrum beta-lactamase; EUCAST: European Committee on Antimicrobial Susceptibility Testing; MALDI-TOF: Matrix-assisted laser desorption localization - time-of-flight; MDRO: Multidrug resistant microorganism; MRSA: Methicillin-resistant

Staphylococcus aureus; VRE: Vancomycin resistant enterococci.

Competing interests

The authors declare that they have no competing interests.

\section{Author's contributions}

MDE, OA, and EP planned and designed the experimental study. MDE, EP LSL, PS, DM, and AW supervised and coordinated data acquisition. MDE and OA performed the statistical analysis. MDE, DM, and OA drafted and revised the manuscript. All authors have participated in analysis and interpretation of data and have read and approved to the final version of the manuscript.

\section{Acknowledgments}

This study was supported by the Medical Scientific Fund of the Mayor of the City of Vienna, Grant-No.: 13010

\section{Author details}

'Department of Hospital Hygiene and Infection Control, Vienna General Hospital, Medical University Vienna, Waehringer Guertel 18-20, 1090 Vienna, Austria. ${ }^{2}$ Division of Clinical Microbiology, Department of Laboratory

Medicine, Medical University Vienna, Vienna, Austria.

Received: 5 October 2014 Accepted: 20 November 2014

Published online: 12 December 2014

\section{References}

1. Pittet D, Hugonnet $S$, Harbarth S, Mourouga P, Sauvan V, Touveneau S, Perneger TV: Effectiveness of a hospital-wide programme to improve compliance with hand hygiene: Infection Control Programme. Lancet 2000, 356:1307-1312.

2. Shiomori T, Miyamoto H, Makishima K: Significance of airborne transmission of methicillin-resistant Staphylococcus aureus in an otolaryngology-head and neck surgery unit. Arch Otolaryngol Head Neck Surg 2001, 127:644-648.

3. Drees M, Snydman DR, Schmid CH, Barefoot L, Hansjosten K, Vue PM, Cronin M, Nasraway SA, Golan Y: Prior environmental contamination increases the risk of acquisition of vancomycin-resistant entrococci. Clin Infect Dis 2008, 46:678-685.

4. Hardy KJ, Oppenheim BA, Gossain S, Gao F, Hawkey PM: A study of the relationship between environmental contamination with methicillinresistant Staphylococcus aureus (MRSA) and patient's acquisition. Infect Control Hosp Epidemiol 2006, 27:127-132.

5. Muzslay M, Moore G, Turton JF, Wilson P: Dissemination of antibiotic-resistant enterococci within the ward environment: the role of airborne bacteria and the risk posed by unrecognized carriers. Am J Infect Control 2013, 41:57-60.

6. Siegel JD, Rhinehart E, Jackson M, Chiarello L, Committee HICPA: Guideline for isolation precautions: preventing transmission of infectious agents in healthcare settings. Am J Infect Control 2007, 2007(35):S65-S164.

7. Kommission für Krankenhaushygiene und Infektionsprävention (KRINKO) beim Robert Koch-Institut (RKI): Hygienemaßnahmen bei Infektionen oder Besiedlung mit multiresistenten gramnegativen Stäbchen. Bundesgesundheitsb/ 2012, 55:1311-1354. 
8. Cooper BS, Stone SP, Kibbler CC: Isolation measures in the hospital management of methicillin resistant Staphylococcus aureus (MRSA): systematic review of the literature. Br Med J 2004, 329:533-539.

9. Gehanno JF, Louvel A, Nouvellon M, Caillard JF, Pestel-Caron M: Aerial dispersal of methicillin-resistant Staphylococcus aureus in hospital rooms by infected or colonised patients. J Hosp Infect 2009, 71:256-262

10. Wigglesworth N, Wilcox MH: Prospective evaluation of hospital isolation room capacity. J Hosp Infect 2006, 63:156-161.

doi:10.1186/s13756-014-0038-z

Cite this article as: Diab-Elschahawi et al:: The strength of coughing may forecast the likelihood of spread of multi-drug resistant microorganisms from the respiratory tract of colonized patients. Antimicrobial Resistance and Infection Control 2014 3:38.

\section{Submit your next manuscript to BioMed Central and take full advantage of:}

- Convenient online submission

- Thorough peer review

- No space constraints or color figure charges

- Immediate publication on acceptance

- Inclusion in PubMed, CAS, Scopus and Google Scholar

- Research which is freely available for redistribution 